\title{
Civilisations
}

Revue internationale d'anthropologie et de sciences

humaines

61-1| 2012

Au-delà du consensus patrimonial

\section{Animaux et objets marrons}

Résistances à la mise en exposition dans les parcs zoologiques et les musées d'Afrique de l'Ouest

\section{Julien Bondaz}

\section{OpenEdition}

\section{Journals}

Édition électronique

URL : http://journals.openedition.org/civilisations/3166

DOI : 10.4000/civilisations.3166

ISSN : 2032-0442

\section{Éditeur}

Institut de sociologie de l'Université Libre de Bruxelles

\section{Édition imprimée}

Date de publication : 22 décembre 2012

Pagination : 121-136

ISBN : 2-87263-038-4

ISSN : 0009-8140

\section{Référence électronique}

Julien Bondaz, «Animaux et objets marrons », Civilisations [En ligne], 61-1 | 2012, mis en ligne le 30 décembre 2015, consulté le 01 mai 2019. URL : http://journals.openedition.org/civilisations/3166 ; DOI : 10.4000/civilisations.3166 


\title{
Animaux et objets marrons \\ Résistances à la mise en exposition dans les parcs zoologiques \\ et les musées d'Afrique de l'Ouest
}

\author{
Julien BONDAZ
}

Résumé : En Afrique de l'Ouest, exposer des animaux sauvages ou des objets rituels ne va pas de soi. Les parcs zoologiques et les musées constituent des espaces ambigus où le pouvoir des bêtes et des choses peut parfois créer du trouble. Les récits d'évasion des animaux de zoo ou les témoignages concernant le déplacement d'objets rituels sont fréquents. Basé sur des données ethnographiques, cet article montre comment animaux et objets sont ainsi parfois dotés de capacités de résistance à leur mise en exposition. On propose alors de parler de marronnage pour décrire cette capacité de résistance, en faisant un retour sur la notion de "vie sociale des objets » et sur l'idée d'anthropomorphisme.

Mots-clés : Afrique de l'Ouest, musée, parc zoologique, exposition, objets rituels, animaux sauvages, anthropomorphisme.

\begin{abstract}
In West Africa, organizing the exhibition of wild animals or ritual objects is far from evident. Zoos and museums are ambiguous places where the power of animals and things can generate some disorder. The narratives concerning the escape of zoo animals or the movement of ritual objects are frequent. Based on ethnographical data, this article shows how animals and objects are sometimes endowed with capacities of resistance against their exhibition. We suggest, then, to speak about marronnage to describe this capacity of resistance, by reexamining the notion of "social life of things" and the idea of anthropomorphism.
\end{abstract}

Keywords: West Africa, museum, zoological garden, exhibition, ritual objects, wild animals, anthropomorphism. 
$\mathrm{E}$

Afrique de l'Ouest, les musées sont généralement peu visités par les populations locales, contrairement aux parcs zoologiques. En guise d'explication de cette faible fréquentation, les professionnels des musées ont souvent recours aux « invocations culturalistes » qui caractérisent certaines « idéologies du développement» (Olivier de Sardan 1995 : 14-15). L'idée selon laquelle les populations locales n'auraient pas la «culture du musée » est ainsi utilisée de manière récurrente - de même que l'idée corollaire selon laquelle « le musée, c'est pour les Blancs ». Lors de mes recherches sur le terrain, un agent du Musée national du Mali m'a par exemple expliqué que « les Maliens en général n'ont pas la culture du musée ${ }^{1}$. Au Musée national du Burkina Faso, la même expression est utilisée : « Les autochtones n'ont pas la culture du musée. Ils ne comprennent pas ». Un artiste nigérien travaillant pour le Musée national Boubou Hama, à Niamey, affirmait pour sa part que «le vrai musée africain, c'est le zoo ». Il est vrai que l'exceptionnel succès populaire du Musée national Boubou Hama du Niger s'explique avant tout par la présence d'un parc zoologique dans son enceinte ${ }^{2}$. Tout se passe donc comme s'il existait une résistance culturelle à la mise en exposition muséale, fondée sur l'opposition entre culture du musée (sous-entendu occidentale) et culture locale (nationale ou africaine). Une telle approche implicitement culturaliste de la question de la fréquentation des musées soulève ainsi de manière frontale celle des résistances aux patrimonialisations. Elle repose sur le postulat selon lequel les résistances au changement traduisent des différences culturelles, voire une dichotomie entre «culture traditionnelle » et « culture moderne », les théories du développement et celles du patrimoine paraissant ainsi relever d'une même « idéologie scientifique » (Olivier de Sardan 2010). Un tel postulat s'explique en partie par la centralité de la question des résistances au changement social dans les approches culturalistes et plus largement par le succès de la notion de résistance en anthropologie, notamment dans les études anglo-saxonnes (Brown 1996).

Une approche anthropologique des résistances aux patrimonialisations ou des controverses patrimoniales doit cependant éviter l'écueil culturaliste, c'est-à-dire l'idée selon laquelle il serait possible d'autonomiser telle ou telle culture et de trouver dans cette autonomie une explication aux actes de résistance. Certes, les faits de résistance relèvent avant tout de stratégies humaines et s'inscrivent donc dans des contextes culturels variés. Les revendications sont souvent locales et mettent en jeu des intérêts privés ou collectifs, traduits en termes politiques. Pour ce qui concerne les musées, la résistance à la mise en exposition de certains objets peut ainsi

1 Les données ethnographiques présentées dans cet article sont issues de recherches menées, pour l'essentiel dans le cadre d'un doctorat en anthropologie, au sein des musées et des parcs zoologiques du Mali (2005-2010), du Niger (2007) et du Burkina Faso (2008). Mon travail consistait alors à observer - chaque fois pendant plusieurs mois - la vie quotidienne et les coulisses de ces institutions, à participer aux différentes missions des agents et à réaliser des entretiens avec eux, ainsi qu'avec des visiteurs et des non-visiteurs (des riverains des musées et des zoos ne les ayant pas visités). Les propos rapportés dans cet article sont (sauf mention contraire) issu de ces divers entretiens.

2 Sur la place des animaux dans ce musée, voir Ibrahim 2012. 
conduire à des débats concernant l'autorité du discours muséal, à des demandes de restitution d'objets par des groupes sociaux, voire à des actes de vandalisme, entre autres « frictions muséales » (Karp et al. 2006). Au sujet des parcs zoologiques, les critiques mettant en cause la capture et l'exposition d'animaux vivants se traduisent le plus souvent par des demandes de remise en liberté ou de réintroduction. Au musée comme au zoo, collectes et mises en exposition sont donc des actions pouvant être discutées, critiquées ou combattues par des humains, qui deviennent ainsi les porteparoles des objets et des animaux concernés. Cet article propose cependant d'inverser la perspective, et de poser l'hypothèse que ces derniers pourraient eux-mêmes être considérés comme entrant en résistance contre leur mise en exposition.

Une telle idée peut sembler curieuse. On imagine assez facilement comment des animaux pourraient ne pas jouer le jeu que leur impose le dispositif du parc zoologique. Les récits d'évasion qui circulent au sujet de certains animaux présentés dans les zoos d'Afrique de l'Ouest paraissent ainsi mettre en scène des actions de résistance animales. De telles actions sont plus difficiles à concevoir de la part d'objets muséaux. L'anthropologie des sciences et des techniques et la sociologie pragmatique nous fournissent certes quelques cas tout à fait intéressants : lorsqu'un objet technique est produit, on ne peut ainsi jamais savoir s'il va « résister, se comporter comme un véritable agent et en retour modifier les actions en cours » (Callon et Law 1997 : 105). Ces questionnements ne sont cependant guère repris au sujet des objets de patrimoine : ce qui résiste dans les patrimonialisations, ce sont toujours des humains. On propose donc ici l'hypothèse selon laquelle l'attribution de capacités de résistance à des non-humains permet de comprendre certains aspects des résistances ordinaires aux patrimonialisations. Deux types de collection a priori distincts, zoologique et muséale, vont nous servir d'exemples. On verra en effet que les questions de mise en exposition d'animaux sauvages et d'objets rituels soulèvent des problèmes parfois similaires dans le contexte ouest-africain. Au Mali, au Niger et au Burkina Faso, de nombreux récits rapportés par des agents ou des visiteurs semblent ainsi perturber ou introduire du jeu dans l'exposition zoologique ou muséale. Ces récits d'évasions d'animaux au zoo et d'évènements provoqués par des objets au musée posent la question de leur mise en exposition et de leur statut. Ils mettent en scène un moment de leur biographie sociale (Appadurai 1986) que l'on propose de nommer « marronnage ». Les récits obtenus révèlent par ailleurs un processus cognitif commun à l'œuvre dans la mise en exposition, une même attribution d'intentionnalité aux animaux de zoo et aux objets de musée, un même anthropomorphisme. Loin de relever de croyances irrationnelles, ils peuvent être entendus comme des récits qui mettent en débat le consensus patrimonial, invitant à une approche intentionnaliste des objets (au sens large) du patrimoine.

\section{Évasions dans les parcs zoologiques d'Afrique de l'Ouest}

\section{S'échapper d'une catégorie instable}

On sait que le statut de l'animal de zoo est particulièrement ambigu : il n'est plus tout à fait sauvage et n'est pas tout à fait domestiqué. L'anthropologue Garry Marvin a ainsi récemment proposé de faire de « l'animal de zoo » une catégorie à part entière 
(Marvin 2008) ${ }^{3}$. Or, les cas d'évasions de ces animaux au statut ambigu mettent en question un tel effort de catégorisation. Comme l'a montré Jean-Pierre Digard (1985), ils inscrivent ces situations dans le cadre plus général du marronnage. Selon lui, les phénomènes de marronnage zoologique ont «beaucoup à nous apprendre sur les hommes qui les produisent ou qui les laissent se produire, sur leurs rapports à la nature et sur leur vision du monde. La déprise trahit l'homme tout autant que l'emprise » (ibid. : 142). Dans ce contexte général, les récits d'évasion apparaissent alors comme des mises en question du zoo envisagé comme espace de domestication.

Dans les discours des visiteurs ou des agents des zoos d'Afrique de l'Ouest, la question du caractère sauvage de l'animal est souvent directement liée à celle de son origine géographique et met en jeu l'opposition entre l'espace urbain et la brousse ${ }^{4}$. Un soigneur m'expliquait ainsi, au sujet des céphalophes de la ménagerie et du zoo du Parc Urbain Bangr Weoogo à Ouagadougou :

Ils sont venus de la brousse et se sont reproduits en ville. Mais comme ils sont d'origine sauvage, on les considère comme sauvages. Mais un animal domestique peut devenir sauvage, s'il s'échappe des mains de l'homme.

Pour lui, la domestication d'un animal sauvage n'est pas possible, alors que l'ensauvagement d'un animal domestique peut se produire accidentellement. En ce sens, les récits d'évasion peuvent parfois servir à argumenter le fait que les animaux sauvages exposés ne sont jamais totalement domestiqués. Ainsi, leur fuite s'accompagne parfois de comportements agressifs interprétés comme des indices de sauvagerie retrouvée. Au Musée national du Niger, par exemple, il y a quelques années, une autruche a été tuée par l'élan du Cap qui s'était échappé de son enclos. Mais les évasions d'animaux ont rarement des conséquences aussi dramatiques. Un gardien de nuit du Musée national du Niger m'a raconté s'être endormi une nuit près des cages des lions, lorsqu'un lionceau en est sorti et a attrapé sa main avec ses pattes de devant. Le gardien se souvient s'être alors réveillé en sursaut et avoir ainsi apeuré le lionceau. De même, un éléphant autrefois exposé dans le même musée avait pour sa part semé la panique au petit marché, sans pour autant faire de victime.

Les récits d'évasion sont cependant avant tout l'occasion de mettre en avant la relation spécifique qui existe entre un animal et son soigneur. Toujours au Musée national du Niger, une femme se souvient ainsi :

Un jour, il y a deux lionceaux qui se sont enfuis du musée, parce qu'à ce moment-là, quand les lionceaux étaient petits, on les laissait se promener dans le musée. Ils sont allés à côté de l'hôtel Gaweye, dans l'herbe haute. Parce que l'endroit était humide, l'herbe était haute. Quand les gens qui passaient sur la

3 Sur ce point, voir également Pellegrini 1995.

4 Le mot français « brousse », issu du vocabulaire colonial, est couramment employé en français d'Afrique et peut s'entendre à différents niveaux. Il est notamment susceptible de désigner à la fois l'espace sauvage par opposition à l'espace villageois, et les zones rurales par opposition aux grands centres urbains. Il renvoie à la fois à « l'autre du village » (Malamoud 1976) et à l'autre de la ville. De tels dualismes doivent cependant être relativisés. L'important ici est que, dans son opposition à l'espace habité, la brousse est décrite comme un espace ambigu et effrayant, peuplé de génies à la fois bons et mauvais et d'animaux sauvages potentiellement dangereux. 
route ont vu leurs têtes dans l'herbe, ils ont eu peur. Ils ont appelé les sapeurs pompiers. Les lionceaux étaient encerclés, ils voulaient les tuer. Heureusement, à ce moment-là, [leur soigneur] est arrivé. Les lions l'ont directement suivi. On aurait dit qu'il savait leur parler. Les gens n'en revenaient pas. Les lions l'ont suivi jusqu'au musée.

Un agent rapporte quant à lui un autre récit d'évasion :

Avant, les chimpanzés étaient attachés à un arbre. Un jour, [une femelle] a cassé ses liens. Elle est allée sur le parking de l'Hôtel de Ville. Je ne sais pas comment elle a fait, elle est entrée dans les voitures, elle a passé les vitesses ou je ne sais quoi, les voitures avançaient et se cognaient. Puis elle est allée dans le marché, il fallait voir ça. Ça a été un vrai scandale. Le musée a dûller chercher [son soigneur] qui était en congé ce jour-là. On a prêté [emprunté] la voiture du musée pour aller le chercher à $100 \mathrm{~km}$ de Niamey.

Dans les deux cas, celui des lionceaux et celui de la femelle chimpanzé, les soigneurs des animaux sont présentés comme ayant un pouvoir sur eux. Dans le premier cas, il apparaît comme leur seul interlocuteur possible (« on aurait dit qu'il savait leur parler »); dans le second, il est présenté comme indispensable à la maîtrise du singe, au point que tous les moyens sont mis en œuvre pour aller le chercher. La proximité entre ces soigneurs et ces animaux montrent que des formes de domesticité existent et résistent à l'évasion, et que le statut de l'animal se caractérise d'abord par une inquiétante instabilités. Les récits d'évasion des animaux ne sont donc pas seulement des manières d'interroger leur sauvagerie ou leur domestication, c'est-àdire le sens que peut prendre leur muséalisation. Ce sont également des moyens de suggérer les relations privilégiées mais complexes que les soigneurs entretiennent avec eux. Extraits de récits de vie des bêtes, ils parlent avant tout des hommes.

On retrouve le même type de récits au parc zoologique de Bamako. On raconte ainsi qu'un lion se serait échappé et continuerait de vivre à côté du zoo, ou qu'un lamantin aurait été tué par un crocodile sorti de son bassin. Récemment, c'est une antilope qui se serait échappée peu après son arrivée au parc et qui aurait réussi à pénétrer jusqu'à l'intérieur du palais présidentiel, situé sur la montagne (Koulouba : « la grande montagne ») qui surplombe le parc zoologique. Entre faits divers et légendes urbaines, ces évasions donnent ainsi à voir le zoo comme un espace potentiellement dangereux, installant la brousse aux portes - voire au cœur - de la ville. Les récits d'évasion bouleversent le principe même du parc zoologique, en mettant à mal la relation à l'animal exposé : l'animal spectaculaire redevient une bête sauvage, refusant en quelque sorte les interactions qui lui sont imposées.

\section{Les récits d'évasion comme signes distinctifs}

Cependant, dans les récits d'évasion, une autre dimension apparaît centrale et renvoie aux représentations que les populations locales se font des animaux. Il

5 Pour une étude de cette proximité entre soigneurs et animaux au zoo et les problèmes que cela soulève, mais dans un autre contexte, voir Estebanez (2010). La notion de « juste place » développée par Isabelle Mauz au sujet des animaux dans les Alpes françaises (Mauz 2002) rend également bien compte du jeu sur la distance entre les humains et les animaux mis en scène au zoo. 
s'agit des relations que les animaux sauvages sont censés entretenir avec des entités invisibles, et notamment des génies de la brousse. L'espace du zoo fonctionne en effet comme un morceau de brousse en pleine ville et sert ainsi d'espace d' " habitation » (Fainzang 1986 : 28) pour des génies de la brousse ${ }^{6}$. Certains animaux sont réputés être eux-mêmes des génies, bien que, selon plusieurs interlocuteurs, les génies prenant une forme animale sont censés ne pas pouvoir être attrapés. Un agent du Musée national du Niger m'expliquait qu'« un génie transformé en animal ne peut pas être capturé : soit il disparaît, soit il se change en autre chose ». C'est ce statut ambigu, non plus entre animal sauvage et animal domestique, mais entre animal et génie, qui expliquerait alors, selon certains, la fréquente disparition des pythons exposés au Musée national du Niger, échappés de leur cage. Ces évasions sont régulièrement interprétées comme des « affaires » de génies, que ce soit sur le mode de la plaisanterie (« c'est sûrement un génie ! ») ou de façon sérieuse. Un agent du musée raconte ainsi :

J'ai entendu un autre bruit qui courait dans la ville. On a perdu trois boas [pythons]. On a dit que c'était les génies qui les avaient fait sortir du trou. Les boas même, c'était des génies, c'est pour ça qu'ils sont sortis.

Un autre, gardien de nuit, témoigne :

De telle heure à telle heure, je ne sais plus quel jour, il paraît qu'il y a un serpent qui sort de l'enclos de l'autruche, qui longe le mur et qui va jusqu'à la grande porte. Un autre gardien dit aussi qu'il l'a vu, et une troisième personne.

La circulation des serpents, leurs évasions fréquentes, trouvent ainsi leur explication dans une relation privilégiée de ces reptiles avec les génies. C'est parce qu'ils ne sont pas des animaux sauvages comme les autres qu'ils peuvent échapper à la captivité et à l'emprise humaine, ou, pour le dire autrement, à leur muséalisation.

Les associations entre certains animaux et certains génies expliquent en outre que les gardiens et les soigneurs qui s'en occupent soient idéalement dotés de compétences magico-rituelles ${ }^{7}$. De manière générale, des dispositions variées, dont le recours à des talismans ou à des médicaments traditionnels, sont d'ailleurs prises par les agents des zoos dans le but de se protéger contre le pouvoir attribué aux animaux ${ }^{8}$. En outre,

6 Pour plus de détails sur ce point, voir Bondaz 2009a. Il convient de noter que le pouvoir « mystique » des animaux varie en degré selon les espèces et selon les individus au sein d'une même espèce. Les animaux «parfaits » ou « monstrueux » (Sperber 1975) en sont les mieux dotés. Toute une série de critères (la taille, l’âge, des indices physiognomoniques tels que la couleur ou la brillance de la robe ou une originalité morphologique...) entrent par ailleurs en ligne de compte, comme le montrent certains exemples développés plus bas. Certains animaux domestiques, notamment le chien et le cheval (notamment parce qu'ils passent de l'espace habité à celui de la brousse), sont également concernés.

7 Par exemple, un responsable du Musée national du Niger insistait sur ce point en ces termes : «La logique veut que tous les animaux soient gardés par des chasseurs ou par les descendants de chasseurs, qui sont dotés d'un pouvoir. Un animal peut s'échapper, mais tu as déjà le pouvoir de le maîtriser, sans agir sur lui ».

8 L'importance des précautions prises peut également s'expliquer, notamment au Mali, par les interdits « totémiques » que certains agents (comme de nombreux visiteurs) continuent de respecter en fonction de traditions familiales. Pour un développement sur ces aspects, voir Bondaz 2010. 
certaines catégories professionnelles sont réputées posséder des compétences magicorituelles permettant de dompter certaines espèces animales. C'est par exemple le cas des forgerons, censés pouvoir charmer les serpents. Les relations entre agents et animaux des parcs zoologiques sont donc susceptibles de s'exprimer en termes de pouvoir magico-religieux. Les logiques d'agression et de protection, de violence et de résistance débordent clairement le cadre du patrimoine. Elles sont d'ailleurs révélées dans des récits plus dramatiques. Ainsi, au parc zoologique de Bamako, un python avait été capturé sur place et s'était échappé dès le lendemain. Décrit comme un " python sacré », il est rendu exemplaire par des traits distinctifs de rareté et de longévité, et, selon certains, sa vision est réputée porter malheur. Plusieurs agents du zoo le rendent responsable du décès d'un gardien, tué par un buffle au début des années 2000. L'un d'entre eux me racontait ainsi :

Quand les pythons vieillissent, certains ont des cornes qui poussent. D'autres changent de couleur et deviennent dangereux. Ce sont des pythons sacrés. Quand on les voit, ça porte malheur. Il y en a un au zoo. Il ne sort qu'une fois par an. Ce n'est pas facile de le voir : il a des siècles! Le gardien qui a été tué par le buffle l'a vu avant de mourir. Dans le parc, avant, il y avait des biches. Il a vu le python attraper une biche et l'a fait échapper. Le buffle était effrayé et l'a attaqué. La femelle a essayé de sauver le monsieur. On l'a amené au point $G$ [nom de l'hôpital] mais soixante-douze heures plus tard, il est mort. Il aurait fallu l'envoyer en Europe, mais on n'avait pas les moyens.

Dans ce récit, le python est rendu directement responsable du comportement du buffle, et donc de l'accident tragique survenu au gardien. La connexion entre les deux événements (le gardien voit le python et le buffle l'attaque) est posée comme une relation causale et sert à construire une image spécifique du python. La proximité avec les animaux sauvages est donc présentée comme un risque, un danger.

À l'inverse, la libération d'un animal du zoo peut parfois être présentée comme largement positive, voire miraculeuse. C'est notamment le cas d'un lion du parc zoologique de Hann, à Dakar, au Sénégal, qui aurait été libéré par Cheick Tidiane Sy, petit-fils du fondateur de la confrérie tijaan. Cheick Tidiane Sy, ambassadeur au Caire, avait été emprisonné dans les années 1960 par le pouvoir senghorien pour une affaire de détournement d'argent public. On raconte qu'après être sorti de prison, il serait passé au parc zoologique de Hann et aurait fait échapper un lion qui est ensuite devenu

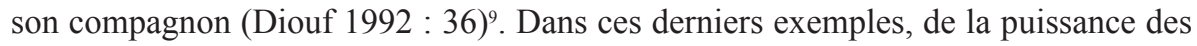
animaux au pouvoir des humains, des génies aux marabouts, les récits d'évasion ou de libération des animaux enfermés en vue de leur exposition mettent ainsi en scène le caractère extraordinaire des acteurs en présence.

La question de la libération des animaux apparaît dans un dernier récit, particulièrement révélateur, qui concerne un lamantin exposé au parc zoologique de Bamako. Le lien souvent établi entre le lamantin et Faro, le génie du fleuve Niger,

9 Cette libération d'un lion du parc zoologique de Hann rejoue une scène importante de la vie du fondateur de la confrérie mouride, Cheick Ahmadou Bamba. On raconte en effet que ce dernier avait réussi à endormir par des prières un lion que les colons français avaient placé dans sa cellule de la prison de Saint-Louis. Les relations entre les deux confréries, tijaan et mouride, sont ici illustrées par la duplication d'un miracle (karamat en wolof) ayant un lion pour sujet central. 
explique que sa présence au zoo soit parfois controversée. Ainsi, en 1961, la capture d'un lamantin dans le Niger et son exposition au zoo coïncident avec des inondations et une crue du fleuve particulièrement dangereuse. Les pouvoirs publics furent contraints d'intervenir et de faire libérer l'animal. Lorsqu'il fut remis à l'eau, Diango Cissé note que « tout rentra dans l'ordre » (1970:13). Le chef de l'État de l'époque, Modibo Keita, aurait été directement concerné par cette affaire, en tant que descendant du premier empereur du Mandé, dont le lamantin est parfois présenté comme l'animal totem, ou tana (Delafosse 1912 : 121 ; Cissé 1970 : 13). Une telle croyance selon laquelle la capture d'un lamantin entraînerait des catastrophes est toujours d'actualité. Ainsi, en 2005, le président Amadou Toumani Touré, en visite dans le delta du Niger, s'est vu offrir deux lamantins vivants par des pêcheurs et les a fait remettre à l'eau après la cérémonie ${ }^{10}$.

Depuis les problèmes de gestion et de sécurité au sein des parcs zoologiques jusqu'aux implications politiques de la mise en exposition d'animaux sauvages, on voit comment les récits d'évasion mettent en question le statut des animaux de zoo. Leur évasion apparaît comme le signe de leur sauvagerie persistante là où tout est mis en œuvre par les soigneurs et les gardiens pour les domestiquer. La transformation des bêtes en objets de patrimoine s'en trouve ainsi perturbée, sinon remise en cause. On va voir qu'en Afrique de l'Ouest, la transformation de certains objets rituels en objets ethnographiques ou en œuvres d'art susceptibles d'être exposés dans un contexte muséal soulève des problèmes souvent proches.

\section{Mouvements d'objets dans les musées d'Afrique de l'Ouest}

Le pouvoir des objets

Dans le contexte de l'Afrique de l'Ouest, de nombreux objets rituels sont considérés comme des « objets forts » (Colleyn 2004 et 2009) : ils sont dotés d'un certain pouvoir magico-religieux. C'est le cas notamment des objets habituellement rassemblés sous l'étiquette de « fétiches ». Or, l'existence de tels objets pose clairement un problème muséologique. La plupart des réflexions sur les musées en Afrique de l'Ouest mettent en avant cette difficulté, ainsi que le nécessaire consensus qu'il faut atteindre au sujet de la mise en exposition de ces objets. Yves Robert écrit par exemple qu' " il s'agit pour le conservateur de musée de dépasser le stade du dilemme entre exposer ou ne pas exposer pour obtenir un consensus acceptable dans le respect des communautés locales et des principes humanistes supérieurs d'éducation » (2007 : 27). Poser la question du pouvoir des objets en termes de consensus empêche cependant de saisir ce que fait le pouvoir attribué à ces objets à leur mise en exposition, et aux visiteurs du musée. Un étudiant malien notait ainsi dans un rapport de stage consacré au Musée national du Mali qu' « une étude approfondie de l'objet s'avère nécessaire pour ne pas exposer les visiteurs aux multiples dangers que peut provoquer la vue d'un objet sacré $\gg{ }^{11}$. Ce n'est donc plus seulement l'objet qui s'expose, ce sont aussi les visiteurs

10 L'Essor, n 15507,8 janvier 2005.

11 Rapport d'étude du milieu, le Musée National de Bamako, Bamako, IPEG, année scolaire 1982$1983: 11$. 
et les agents du musée qui s'exposent à l'objet. Le pouvoir attribué à certains objets rituels soulève ainsi le problème du danger de leur mise en exposition et explique la nécessité de leur faire subir un traitement rituel avant leur entrée dans les collections ${ }^{12}$. L'un des guides du Musée national du Mali explique par exemple, à propos des objets rituels :

Avant de le donner au musée, on le désacralise. Parce qu'il y a trois modes d'acquisition : achat, don, legs. S'il s'agit d'un achat, le vendeur le désacralise, dans les fins fonds, il le désacralise chez lui avant de le vendre au musée. Si c'est [un] don, c'est la même chose ; si c'est [un] legs, c'est la même chose. Dans les trois cas, le propriétaire est obligé de désacraliser l'objet à la base, avant de le remettre au musée.

Mais certains objets sont considérés comme trop puissants pour perdre tout leur pouvoir : malgré les rituels de désacralisation effectués lors de la collecte des « objets forts ", le pouvoir de certains d'entre eux reste dangereux pour les gardiens de salle ou pour les visiteurs. Un professeur de socio-anthropologie rencontré à l'Institut des Sciences Humaines de Bamako m'expliquait ainsi, au sujet du transfert d'objets rituels dans un musée : «Du sacré au profane, le cap n'est pas facile à passer. Il y a toujours des restes ». Toujours au Mali, à Samako, sur la route de Sibi, un bolitigi (« féticheur») m'a tenu un discours identique à propos de ses propres boliw (« fétiches ») : « Mes boliw sont tellement puissants qu'ils ne pourront jamais aller au musée. Tout le monde ne peut pas les voir. Ils ont tellement de nyama que je ne pourrai jamais enlever tout le nyama ». Cette idée selon laquelle il reste toujours un certain pouvoir aux objets rituels réputés les plus puissants est largement répandue et apparaît centrale dans les enjeux liés à la constitution d'un patrimoine de collection en Afrique de l'Ouest.

Au Musée national du Mali, trois objets en particulier sont censés continuer à exercer un certain pouvoir : un Komokun, un Poro et un boli. L'un des gardiens de la salle des « Chefs d'œuvre d'art rituel » explique par exemple :
Si un komotigi ["maître du Komo ", c'est-à-dire responsable du culte du Komo] vient et s'adresse au Komokun, la vitrine va exploser. C'est la même chose pour le boli. Si un bolitigi [" maître du boli", habituellement traduit par «féticheur»] vient et parle au boli, le nyama va revenir dans le boli et la vitrine va exploser.

On peut d'ailleurs noter que, dans cette manière de dire la puissance contenue de l'objet (son nyama), on voit comment le principe du musée est mis en cause, la destruction de la vitrine pouvant à cet égard paraître symptomatique. Au Musée national du Mali, comme dans d'autres musées d'Afrique de l'Ouest, des récits circulent par ailleurs au sujet de certains objets susceptibles de provoquer la folie, la maladie ou la mort de leurs gardiens. Au Musée national du Niger, des objets utilisés

12 Les agents des musées concernés parlent souvent de « désacralisation » à ce sujet. Il me semble cependant qu'une telle notion demeure problématique dans le contexte ouest-africain, où la démarcation entre sacré et profane n'est pas toujours opératoire. C'est donc avant tout par commodité que nous reprenons le terme dans la suite de cet article. 
dans le cadre des cultes de possession qui provoquaient l'entrée en transe de certains visiteurs ont été retirés de l'exposition (Bondaz 2009b) ${ }^{13}$.

Dans un tel contexte, plus encore que la vue des objets, le contact des visiteurs avec eux peut s'avérer particulièrement dangereux. Au sein des musées d'Afrique de l'Ouest, l'interdiction de toucher les objets trouve ainsi un nouveau sens. Dans certains cas, en effet, ce n'est pas le souci de la conservation des objets qui motive l'interdit, mais celui de la protection des personnes. À Ouagadougou, au Burkina Faso, un agent du Musée de la Musique explique par exemple :

Il y a des objets qu'on interdit de toucher. Si un visiteur touche certains objets, il peut tomber malade ou s'évanouir. On ne lui explique pas. On dit qu'il a eu un malaise, mais c'est parce qu'il y a des objets qu'il ne faut pas toucher.

Au Musée national du Burkina Faso, de telles remarques sont régulièrement émises par les guides lors des visites, notamment scolaires, et entretiennent ainsi un jeu sur la peur ressentie par les visiteurs à la vue de certains masques exposés. Les touristes sont eux aussi susceptibles d'être pris dans un tel discours. Un agent du musée témoigne ainsi : " Il y avait un guide, quand il y avait des visiteurs blancs, il s'amusait à leur dire que les masques marchaient la nuit : ça faisait peur aux Blancs et ils regardaient les masques avec plus d'attention ». Le contact avec les objets ou le déplacement des masques constituent ainsi des motifs saillants du discours des guides, et entretiennent l'ambiguïté entre la conscience du danger de l'exposition aux objets et le jeu de sidération que cela peut provoquer chez les visiteurs, entre mesure de sécurité et « technologie de l'enchantement» (Gell 1992 ; Wastiau 2008).

\section{Échapper à l'exposition muséale}

Le pouvoir dont sont dotés certains objets rituels exposés dans un musée se manifeste également, dans les récits, par l'attribution de capacités de mouvement, de déplacement. A Ouagadougou, on raconte par exemple qu'un timbouani (tambour double bwaba) a dû être retiré de l'exposition, que ce soit au Musée de la Musique ou au Musée national :

On a eu des problèmes. Des choses bizarres se sont passées. (un agent du Musée de la Musique)

Avant, il y avait un tambour qui était exposé, chaque fois il bougeait. Quand on venait, on le trouvait trois mètres plus loin, collé à un autre objet. (un gardien du Musée national)

Plusieurs autres objets sont également réputés se déplacer ou faire du bruit. Deux femmes qui travaillent au Musée national évoquent des souvenirs récents :

13 Dans les musées d'Afrique de l'Ouest, il est habituel de laisser ou de remiser les objets réputés les plus puissants dans les réserves des musées (on en trouvera plus bas un autre exemple). Les personnes qui pénètrent dans les réserves (conservateurs ou gardiens) se voient d'ailleurs souvent attribuer des compétences magico-rituelles ou des moyens de protection spécifiques par leurs collègues. 
Avant, la nuit, il y avait du bruit dans la salle des masques. On entendait marcher. Je ne sais pas s'ils ont fait quelque chose ou s'ils ont enlevé le masque, mais maintenant, c'est fini.

La nuit, dans la salle des masques, ça tourne.

Une troisième femme rapporte un souvenir plus lointain ;

Quand il y avait une salle d'exposition à l'hôtel Indépendance, il y avait une statuette mossi qui marchait pendant la nuit. Chaque nuit, les policiers en faction devant l'hôtel entendaient du bruit. C'était une statuette qui faisait peur. Même si elle avait été désacralisée, ses effets continuaient. Sa puissance était si grande que ses effets ont mis du temps à partir.

Cette statuette de protection familiale (tilembugri) autrefois exposée à l'hôtel Indépendance est en fait toujours présente dans les collections du musée. Elle a récemment été placée à l'entrée du pavillon Jean Rouch, lors de l'exposition «Chefs d'œuvre d'art rituel du Musée National », où elle se serait également, selon un agent, déplacée pendant la nuit : «Quand on la posait là, le lendemain on la retrouvait ici. Il a fallu la ramener à Ouahigouya pour faire des sacrifices. Tous les gardiens de nuit se plaignaient ». La statuette a ensuite été placée dans la réserve, où elle se trouve encore aujourd'hui et où elle aurait continué à perturber la vie du musée, comme me l'a rapporté un autre agent du musée :

Dans la réserve, il y avait une statuette qu'on avait amenée de Ouahigouya, avec des cauris sur la tête et quelque chose [il fait le geste : autour des hanches]. C'était dur. Vraiment c'était dur. On l'enfermait le soir. Le matin elle était dehors. On l'a ramené à Ouahigouya pour qu'ils diminuent sa puissance. Maintenant ça va, elle est toujours dans la réserve, mais c'est calme.

Seul un traitement rituel spécifique (nécessitant un retour dans son contexte rituel d'origine) a donc finalement pu venir à bout de la puissance de la statuette. C'est d'ailleurs l'objet qui a été choisi comme logo du Musée national. L'un des responsables du musée explique ce choix en se référant à la petite taille de la statuette et à sa fonction protectrice : «Le musée est petit mais va grandissant. Le musée est protégé et va s'épanouir en grandissant ». L'histoire d'un tel emblème semble ainsi (implicitement) indiquer les difficultés rencontrées par les conservateurs du musée dans la transformation de certains objets rituels en objets muséaux. Le fait que cet objet soit désormais mis en réserve peut en ce sens paraître révélateur.

La question du pouvoir des objets dépasse cependant le cadre national. Toujours au Musée national du Burkina Faso, des objets prêtés par le musée pour des expositions organisées en Europe sont également réputés avoir eu des comportements étranges, presque humains :

Il y avait une statue qu'on avait envoyé exposer en France, elle n'arrêtait pas de pleurer. Chaque matin, on la trouvait avec des larmes qui avaient coulé de ses yeux. Le pays lui manquait. Elle était triste là où elle était.

Un masque du musée avait été prêté pour une exposition en France. Un matin, on l'a trouvé au musée. Il était revenu seul... [Après un silence] Il a voyagé sans billet. 
On retrouve ces histoires à propos d'objets volés exposés en Europe, par exemple, au Mali, à propos de masques dogon ${ }^{14}$ :

Dans les années 1970, des voleurs avaient pris un masque dogon caché dans une grotte, dans la falaise. Le masque avait été revendu, et d'intermédiaire en intermédiaire, il était arrivé jusque dans la collection d'un Français ou d'un Belge, je ne sais plus. Mais peu de temps après, le masque est revenu. Un jour, il est revenu tout seul dans la grotte. Il avait fait tout le chemin à l'envers. Et tous les vendeurs, et les voleurs, tous sont morts. Le masque les a tués.

Il s'agit ainsi de mettre en récit le danger que constitue la transformation d'objets rituels en patrimoine de collection, à travers l'évocation du décès des intermédiaires. Plus globalement, ce ne sont plus seulement des enjeux locaux qui sont mis en avant, mais bien l'articulation entre les représentations et les savoirs locaux concernant les objets rituels et leur circulation internationale. Depuis la capacité attribuée à certains objets de faire exploser leur vitrine à celle de revenir dans leur musée ou leur contexte d'origine, les récits qui sont transmis au sein des musées d'Afrique de l'Ouest montrent que la mise en exposition des objets rituels a quelque chose à voir avec celle des animaux sauvages. Parmi les uns comme parmi les autres, certains objets rituels et certains animaux sauvages sont dotés d'une capacité à lutter contre leur mise en vitrine ou leur mise en cage, c'est-à-dire à échapper au contexte muséal et au style d'interactions qui est produit par leur mise en exposition.

\section{Le marronnage des animaux et des objets comme résistance à la mise en exposition}

Il est désormais temps de s'interroger sur ce qui est en jeu dans ces récits, et qui nous intéresse ici : la question de la transformation difficile et parfois dangereuse de certains animaux sauvages et de certains objets rituels en patrimoine de collection, c'est-à-dire la question de leur requalification en objets de patrimoine. Leur mise en exposition constitue en effet une étape essentielle de leur biographie et il importe de revenir ici sur la proposition faite par Arjun Appadurai (1986), de considérer la vie sociale des objets et de mettre en place une méthode biographique pour l'analyse de la culture matérielle. Une telle méthode se révèle particulièrement heuristique lorsqu'il s'agit d'étudier les patrimonialisations et, en particulier, pour ce qui nous concerne, la muséalisation de certains objets. On pense notamment aux travaux de Thierry Bonnot (2002, 2004) ou aux réflexions de Fabrice Grognet (2005). On pense surtout, pour ce qui concerne le contexte africain, aux analyses de Boris Wastiau $(2000,2004)^{15}$. Il me semble que cette méthode biographique mériterait d'être appliquée plus souvent aux animaux. C'est du moins l'une des propositions de cet article. Mais surtout, une

14 La plupart des récits rapportés dans ce texte sont en effet proches des récits de chasse ou de vol d'objets rituels en contexte ouest-africain : les difficultés, les échecs ou les maladies des chasseurs et des voleurs trouvent une explication dans le pouvoir ordinairement attribué (toujours selon des degrés divers) aux animaux sauvages et aux « objets forts ». De ce point de vue, un parallèle existe entre la chasse ou le vol et la mise en exposition.

15 On peut également mentionner le numéro du Journal des Africanistes consacré à la vie sociale des objets dans les sociétés du Sahara (2006). 
telle méthode soulève plusieurs interrogations, en particulier au sujet de l'usage métaphorique des notions de vie et de mort et de la comparaison entre esclave et objet que propose Igor Kopytoff dans l'ouvrage d'Appadurai (Kopytoff 1986). On se souvient que Kopytoff établit un parallèle entre l'esclavage envisagé comme « processus de transformation sociale » (65) et la « biographie économique » des objets comprise comme attribution aux marchandises (commodities) de différents sens, selon des processus constants de reclassification et de requalification. Pour Igor Kopytoff, ce parallèle est valable pour tous les objets, en particulier pour les œuvres d'art (67), et permet de mettre en évidence le fait qu'un objet n'a pas de valeur, de statut ou de signification intrinsèque ou immuable. Un tel rapprochement peut pourtant être critiqué dans la mesure où il pose le problème de la chosification des êtres humains en même temps qu'il propose d'anthropomorphiser les objets (Bondaz 2008).

C'est cependant une autre critique qui va nous retenir ici. On peut en effet s'étonner que, lorsque Kopytoff propose de comparer la biographie des choses avec celle des esclaves, il ne mentionne pas le cas du marronnage à ce propos. La comparaison entre la biographie des objets et celle des esclaves relève de l'exercice métaphorique. La métaphore permet en effet « de comprendre quelque chose (et d'en faire l'expérience) en termes de quelque chose d'autre » (Lakoff et Johnson $1985: 15$ ) et, de ce point de vue, la question du marronnage se révèle tout à fait intéressante. Le marronnage perturbe la linéarité de la biographie de l'esclave. Il constitue en effet un acte de rébellion ou de résistance et l'on ne s'étonnera donc pas si la figure de l'esclave marron a pris toute son importance dans l'anthropologie culturaliste, autour de la question de la résistance culturelle. Comme l'exprime Rafaël Lucas, le marronnage apparaît comme une « résistance à la dynamique de brouillage ontologique que contient l'esclavage » (Lucas 2002). Or, on sait que le terme de «marronnage » appliqué aux esclaves vient de son usage au sujet des animaux ${ }^{16}$. Il s'agit dans un cas de retourner à la liberté, dans l'autre, comme on l'a vu à propos des animaux évadés de zoos, de retourner à la vie sauvage. Dans certains récits concernant les objets rituels mis en exposition, le même type de processus paraît être à l'œuvre : certains d'entre eux résistent à la mise en exposition et on attribue même aux objets réputés les plus puissants des capacités de mouvement ou de fuite.

Les récits de marronnage d'objets et d'animaux invitent dès lors à une approche intentionnaliste de ces non-humains (Freedberg 1989 ; Schaeffer 1996 et 2004 ; Gell 1998 ; Descola 2006 et 2009). Ces récits reposent en effet sur un principe commun : l'attribution d'intentions ou, pour le dire autrement, l'anthropomorphisme. La distinction que propose Stewart Guthrie entre l'anthropomorphisme ( «l'attribution de caractéristiques humaines à des événements et à des choses qui ne sont pas humains ») et l'animisme ("l'attribution de vie à ce qui est sans vie ») apparaît en effet précieuse (Guthrie 1989 : 39). Tandis que les animaux des zoos sont anthropomorphisés ${ }^{17}$, pour ce qui concerne les objets de musée, anthropomorphisme et animisme peuvent se

16 Voir l'étymologie et l'historique du mot dans Rey 1992.

17 Sur l'anthropomorphisation des animaux de zoo, voir Mullan et Marvin 1999 : chapitre 1. Pour une synthèse des réflexions sur l'anthropomorphisme animal, on peut par exemple se reporter à Renk et Servais 2002 : chapitre 11 . 
rejoindre lorsque certains « objets forts » sont réputés se déplacer la nuit ou voyager par leurs propres moyens. Dans les deux cas, nous avons affaire à des non-humains qui sont définis comme des agents intentionnels. Nous pouvons ainsi poser la question de la résistance en de nouveaux termes. En effet, non seulement les récits de marronnages d'objets et d'animaux reposent sur un principe anthropomorphe, mais les intentions attribuées aux objets et aux animaux apparaissent comme des intentions d'échapper au contexte muséal, et donc de résister à la patrimonialisation. Tout se passe comme si les humains déléguaient aux non-humains une certaine intention de résister à la mise en exposition.

En Afrique de l'Ouest, certains animaux sauvages et certains objets rituels, souvent associés à des entités invisibles, se retrouvent dotés de pouvoirs et d'intentions de résister à leur mise en cage ou en vitrine. Animaux et objets marrons démontrent ainsi que, pour penser les patrimonialisations, il ne s'agit pas seulement de prendre en compte le point de vue institutionnel, ni même le point de vue des humains en général, mais également (si l'on peut dire) celui des non-humains. Ces récits invitent ainsi à réfléchir aux manières qu'ont les humains d'attribuer ou de déléguer des intentionnalités aux non-humains au sujet de leur patrimonialisation. Ce faisant, nous évitons l'écueil du culturalisme dans les réflexions concernant les résistances aux patrimonialisations, mais nous n'abandonnons pas pour autant une approche anthropologique de ces patrimonialisations, puisque les manières d'attribuer des intentions aux animaux et aux objets varient en fonction des contextes culturels, comme nous avons pu le voir à propos de l'Afrique de l'Ouest. Nous remplaçons l'explication culturaliste par l'étude des interactions entre humains et non-humains. Les animaux et les objets marrons obligent ainsi à agencer d'une nouvelle manière non seulement les actes de résistance, mais aussi les projets de patrimonialisation et l'étude anthropologique que l'on peut en faire. Appliquer la « méthode biographique » à l'étude des animaux de zoo et des objets de musée peut en effet permettre de mieux comprendre comment ils sont eux aussi les agents (parfois résistants) de leur mise en patrimoine.

\section{Références citées}

Appadurai, Arjun (dir.), 2003 [1986]. The Social Life of Things. Commodities in Cultural Perspectives. Cambridge : Cambridge University Press.

Bondaz, Julien, 2008. « Mort de l'objet et vif du sujet. Des fétiches au Musée National du Mali », in Michèle Cros et Julien Bonhomme, Déjouer la mort en Afrique. Or, orphelin, fantômes, trophées et fétiches, 129-158. Paris : L'Harmattan.

-, 2009a. « Topographie magico-religieuse et espace muséal. Territoires, trajectoires et transes au Musée National du Niger », in Etienne Berthold (dir.), Patrimoine et sacralisation, patrimonialisation $d u$ sacré, 269-282. Québec : éditions Multimondes.

-, 2009b. « Le génie du lieu. La fusion des patrimoines matériels et immatériels au Musée National du Niger », in Laurier Turgeon (dir.), Spirit of Place : Between Tangible and Intangible Heritage. L'esprit du lieu : entre le patrimoine matériel et immatériel, 23-32. Québec : Presses de l’Université Laval.

-, 2010. « La maison où voir le lion. Pistes fauves au zoo de Bamako (Mali) », in Michèle Cros et Julien Bondaz (textes rassemblés et présentés par), Sur la piste du lion. Safaris ethnographiques entre images locales et imaginaire global, 107-118. Paris : L'Harmattan.

Bonnot, Thierry, 2002. La vie des objets. Paris : éditions de la Maison des sciences de l'homme. 
-, 2004. « Itinéraire biographique d'une bouteille de cidre », L'Homme 170 : 139-164.

Brown, Michael Fobes, 1996. « On Resisting Resistance », American Anthropologist 98 : 728-735.

Callon, Michel, et John Law, 1997. «L'irruption des non-humains dans les sciences humaines », in Bénédicte Reynaud (dir.), Les limites de la rationalité. Les figures du collectif, 99-118. Paris : La Découverte.

Cissé, Diango, 1970. Structures des Malinké de Kita. Bamako, Editions populaires.

Colleyn, Jean-Paul, 2004. « L'alliance, le dieu, l'objet», L'Homme 170 : 61-78.

-, 2009. « Images, signes, fétiches. À propos de l'art bamana (Mali) », Cahiers d'Études Africaines 195 : $33-746$.

Delafosse, Maurice, 1912. Le Haut-Sénégal-Niger, tome 1. Paris : Larose.

Descola, Philippe, 2006. « La fabrique des images », Anthropologie et sociétés 30 (3) : 167-182.

-, 2009. «L'Envers du visible : ontologie et iconologie », Histoire de l'art et anthropologie. Paris : coédition INHA-Musée du quai Branly («Les actes »), http://actesbranly.revues.org/181]

Digard, Jean-Pierre, 1995. "Un phénomène méconnu : le marronnage. Aspects modernes et implications », in Bernadette Lizet et Georges Ravis-Giordani (textes réunis par), Des bêtes et des hommes. Le rapport à l'animal : un jeu sur la distance, 133-145. Paris : Editions du C.T.H.S.

Diouf, Mamadou, 1992. «Islam : Peinture sous verre et idéologie populaire », in Bogumil Jewsiewicki (éd.), Art pictural zaïrois, 29-40. Sillery : Editions du Septentrion.

Estebanez, Jean, 2010. « Ceux qui sont proches : les soigneurs au zoo », Société 108 (2) : 47-57.

Fainzang, Sylvie, 1986. "L'intérieur des choses ». Maladie, divination et reproduction sociale chez les Bisa du Burkina. Paris : L'Harmattan.

Freedberg, David, 1988. Le pouvoir des images. Paris : Gérard Monfort éditeur.

Gell, Alfred, 1992. "The technology of enchantment and the enchantment of technology », in Jeremy Coote et Anthony Shelton (dir.), Anthropology, Art and Aesthetics, 40-63. Oxford : Clarendon Press.

-, 1998. Art and Agency. An anthropological Theory. Oxford : Clarendon Press.

Grognet, Fabrice, 2005. « Objets de musée, n’avez-vous qu’une vie ? », Gradhiva 2 (n.s) : 49-63.

Guthrie, Stewart, 1993. Faces in the Clouds. A New Theory of Religion. New York : Oxford University Press.

Ibrahim, Mamane, 2012. «L'exposition des animaux vivants au Musée National Boubou Hama de Niamey (Niger) », in Michèle Cros, Julien Bondaz et Maxime Michaud (éds), L'animal cannibalisé. Festins d'Afrique, 127-139. Paris : Éditions des Archives Contemporaines.

Karp, Ivan, Corinne A. Kratz, Lynn Szwaja et Tomás Ybarra-Frausto (éds), 2006. Museum Frictions : Public Cultures/Global Transformations. Durham-London : Duke University Press.

Kopytoff, Igor, 2003 [1986]. " The cultural biography of things : commodization as process », in Arjun Appadurai (dir.), The social life of Things. Commodities in cultural perspectives, 64-91. Cambridge : Cambridge University Press.

Lucas, Rafael, 2002. «Marronnage et marronnages », Cahiers d'histoire. Revue d'histoire critique 89 : 13-28.

Malamoud, Charles, 1976. "Village et forêt dans l'idéologie de l'Inde brahmanique », Archives européennes de sociologie XVII (1) : 3-20.

Malé, Salia, 1999. «La présentation des objets de culture vivante : le cas du Musée National du Mali », Journal des africanistes 6 (1) : 29-52. 
Marvin, Garry, 2008. «L'animal de zoo. Un rôle entre sauvage et domestique », Techniques et culture $50: 102-119$.

Mauz, Isabelle, 2002. «La conception de la juste place des animaux dans les Alpes françaises », Espaces et sociétés 110-111 (3-4) : 129-145.

Mullan, Bob et Garry Marvin, 1999 [1987]. Zoo Culture. Urbana and Chicago : University of Illinois Press.

Olivier de Sardan, Jean-Pierre, 1995. Anthropologie et développement. Essai en socio-anthropologie du changement social. Paris : APAD-Karthala.

-, 2010. « Le culturalisme traditionaliste africaniste. Analyse d'une idéologie scientifique », Cahiers d'Études africaines 198-199-200 : 419-453.

Pellegrini, Patricia, 1995. «Zoos, parcs et réserves, quel est le statut de ces animaux offerts au regard de l'homme? ", in Bernadette Lizet et Georges Ravis-Giordani (textes réunis par), Des bêtes et des hommes. Le rapport à l'animal : un jeu sur la distance, 227-242. Paris : Éditions du C.T.H.S.

Renck, Jean-Luc, et Véronique Servais, 2002. L'éthologie. Histoire naturelle du comportement. Paris : Seuil.

Rey, Alain (dir.), 1992. Dictionnaire historique de la langue française. Paris : Le Robert.

Robert, Yves, 2007. « Musées en Afrique entre universalité et singularité : réflexions et propositions », in Anne-Marie Bouttiaux (textes réunis par), Afrique : musées et patrimoines pour quels publics?, 13-33. Tervuren-Paris : Musée royal de l'Afrique centrale-Karthala-Culture Lab Éditions.

Schaeffer, Jean-Marie, 1996. Les célibataires de l'art. Pour une esthétique sans mythes. Paris : Gallimard.

-, 2004. «Objets esthétiques », L'Homme $170:$ 25-45.

Sperber, Dan, 1975. " Pourquoi les animaux parfaits, les hybrides et les monstres sont-ils bons à penser symboliquement? », L'Homme 15 (2) : 5-34.

Tardieu, Jean-Pierre, 2006. "Cimarrón-Maroon-Marron. Note épistémologique », Outre-Mers t. 94, $350-351: 237-247$.

Wastiau, Boris, 2000. ExitCongoMuseum. Un essai sur la «vie sociale » des chefs d'œuvre du musée de Tervuren. Tervuren : Musée royal de l'Afrique centrale.

-, 2004. « From Mpala to Tervuren : the ritual Life of two Congolese Masterpieces of the Royal Museum for Central Africa, 1880-2000 », in Mary Bouquet et Nuno Porto (dir.), Science, Magic and Religion. The Ritual Processes of Museum Magic, 95-115. Oxford : Berghahn.

-, 2008. Medusa en Afrique. La sculpture de l'enchantement. Genève : 5 Continents-Musée d'ethnographie de Genève. 